\title{
Assessing Differing Eyelash Extension Compositions and their Microorganisms
}

\author{
Medha Tripathi ${ }^{1}$
}

${ }^{1}$ Athens High School, Troy, MI, USA

\section{ABSTRACT}

The purpose of this study was to determine the quantitative relationship between parasite Demodex folliculorum and three different eyelash extension types: mink, sable and silk. In spite of the disruption of experimentation and the resulting necessary altercations due to the COVID-19 pandemic, the research method followed was quasi-experimental. Rather than directly assessing the correlation between eyelash extension types and Demodex folliculorum, the relationship both variables had with bacteria Escherichia coli and Staphylococcus epidermidis was examined to reach a conclusion. The process of experimenting and synthesizing prior information resulted in heavy usage of primary and secondary data to reach the conclusion that sable eyelash extensions comparatively promote the least growth of Demodex folliculorum and are therefore the safest option for eyelash extension users.

\section{Introduction}

As the cosmetics industry expands the scope and complexity of the products it develops, a product known as eyelash extensions has taken the center stage as the new yearned commodity in the beauty community. Eyelash extensions are a new cosmetic asset that work to enhance the natural eyelashes of the user to become longer, fuller, thicker, and increase the overall attractiveness of natural eyelashes. According to Andra Kwartang, artistic director and expert lash stylist at Courtney Akai Lash Boutique in NYC, eyelash extensions "are applied by a technician who hand-glues the extensions on top of your natural lashes." Apart from their natural appearance, eyelash extensions are appealing to women because of their durability: anywhere ranging from 4-8 weeks. ${ }^{10}$

The different groupings of eyelash extensions allow consumers to carefully select each characteristic they desire their eyelash extensions to have. Both the technician and the consumer must first take into consideration fixed factors such as eye shape, size of eyes, type of eyelids, orientation of eyes, and qualities of natural eyelashes when determining the eyelash extensions that are most suitable for the potential wearer. Once these factors have been acknowledged, the consumer must select the material, length, curve, and color of their eyelash extensions. The largest classification of the aforementioned categories is the composition, or material, of eyelash extensions. The three main types of eyelash extensions are mink eyelash extensions, sable eyelash extensions, and silk eyelash extensions. ${ }^{27}$

Eyelash extensions are sold as individual fibers rather than a strip, so they are held together and attached to the natural eyelashes with the usage of glue specifically manufactured for eyelash extensions. Dermatologists Sangeetha Shanmugam and Mark Wilkinson conducted research exploring the ingredients and structure of various eyelash extension glues and what the implications of this may be. Shanmugan and Wilkinson "found high formaldehyde emission rates in all three types of [eyelash extension] glue" as well as Cyanoacrylates, which "are widely used commercially as allpurpose "super glues". ${ }^{2}$ According to Michigan State University, Formaldehyde is an organic structure that falls under the functional group of aldehydes, and aldehydes are characteristically known to consist of a -CHO structure with a carbonyl center, which is attached to any alkyl or side chain group. Carbon is the basic element of life, and because of its presence in formaldehyde, bacteria is attracted to the formaldehyde. ${ }^{22}$ The formaldehyde in eyelash extensions thereby increases the accumulation of bacteria, and because consumers are often worried about the 
fragility of their eyelash extensions, they do not properly clean the eyelash extensions, leading to even more bacterial infestation.

The threats posed by eyelash extensions to human health are often overlooked, despite their detrimental effects. Healthline Media, Inc., an American health information provider, lists several of these effects: "bloodshot eyes, burning, itching, pain, rash, redness and inflammation of the eye or eyelid, swelling," the aforementioned infections due to the formaldehyde, and loss of eyelashes. ${ }^{28}$ All of these side effects have been long known and are associated with makeup products similar to eyelash extensions, such as mascara and fake eyelashes. However, one of the most prevalent health concerns regarding eye cosmetics is the growth of Demodex folliculorum in eyelash extensions. ${ }^{9}$

Demodex Folliculorum is a parasite that lives on the hair follicles and dead skin cells humans. These parasites are between 0.3-0.4 mm, have four pairs of legs, and generally have a lifespan ranging from 2-3 weeks. ${ }^{8}$ Eyelash extensions and Demodex Folliculorum are often considered analogous with hair and head lice in the sense that Demodex folliculorum is a parasite that can cause extreme itching to the victim of the infestation. ${ }^{28}$ In addition, ophthalmologist Jade Coats finds that Demodex folliculorum can cause several other health problems such as "red and swollen lid margins, madarosis, chronic MGD, and chronic blepharoconjunctivitis". ${ }^{9}$ This parasite and its relationship to the types of eyelash extensions and bacteria will be the focus of the research conducted in this paper. Bacterium that have been found to use Demodex folliculorum as a bacterial vector, or as DNA that can store foreign genetic material, are Bacillus oleronius and Staphylococcus epidermidis.

Bacillus oleronius is a gram-negative rod-shaped bacterium with the thick peptidoglycan structure of a grampositive bacterium, making Bacillus oleronius a unique bacterium. ${ }^{21}$ In a study conducted by professors Noreen Lacey, Kevin Kavanagh and Scheffer C.G. Tseng from National University of Ireland, Bacillus epidermidis was isolated from the Demodex folliculorum found on eyelashes. Through this experiment it was found that "co-morbidity between [Demodex] mites and Bacillus [oleronius] rests in the symbiosis of the latter in the former". ${ }^{11}$ In simple terms, a positive correlation exists between Demodex folliculorum and Bacillus oleronius, and Demodex folliculorum often carries this bacterium within it.

Staphylococcus epidermidis is a gram-positive spherically shaped facultative anaerobic bacterium that is often on skin flora and follicles of humans, similarly to Demodex folliculorum. ${ }^{20}$ The bacterium is $0.5-1.5$ um, which is exceptionally small, and Staphylococcus epidermidis forms in clusters, yet another characteristic that it shares with Demodex folliculorum. Optometrist Chester H. Pheiffer conducted an experiment where he observed the eyelashes of patients that had Demodex folliculorum. Of the people he observed, "43 persons from whose lid margins Staphylococcus... was cultured seemed to have been more likely to have two or more mites than those without." ${ }^{7}$ Once again, the connection between a pathogen, in this case Staphylococcus epidermidis, and Demodex folliculorum is undeniable.

Demodex folliculorum is an extremely common parasite among humans. In fact, after crossing the age of forty, humans are naturally prone to Demodex folliculorum and most likely have the parasite unless they are taking active measures against it. ${ }^{9}$ The most common area for Demodex to reside in are the eyelashes, and Berkeley graduate and optometrist Tanya Gill claims that along with the increased use of eyelash extensions there has been "a surge in eyelash extension related infections." ${ }^{26}$ In fact, many of the symptoms to humans that were characteristically affiliated with eyelash extensions are also common to Demodex folliculorum, such as irritation and redness.

Due to the discovery of the newfound correlation between eyelash extensions and Demodex folliculorum, there is a current gap in the body of knowledge that concerns how the composition of the different types of eyelash extensions in the market affect the presence of Demodex folliculorum in the eyelash extensions. One may therefore inquire: "How does the composition of differing eyelash extension types impact the growth of bacterium that ultimately promotes the increase of the parasite Demodex folliculorum near the eye?" This study attempts to determine whether mink, sable, or silk eyelash extensions is the least prone to growing bacteria that will eventually stimulate the infestation of Demodex folliculorum on the eyes and how the variances in the composition of the differing eyelash extensions leads to the yielded bacterial growth. For the purposes of this research, the shape, size, and orientation of the tested eyelash extensions the same. This is to ensure that the produced results are not affected by the variables listed, but rather solely the composition of the hair-resembling follicles that are used to compose eyelash extensions. 
Eyelashes prevent harmful particles from entering the eye, one of the most sensitive human parts. Therefore, when a cosmetic is supplementing a body part as integral as eyelashes, users of eyelash extensions must be extremely cautious for the safety of their eyes. The findings of this research topic is hence important because it will serve to inform eyelash extension users of which type of eyelash extensions they should wear in order to prevent the growth of Demodex folliculorum in their eyelash extensions as well as maintain overall health and hygiene around their eyes.

\section{Review of Literature}

In order to understand why this study analyzes the relation between eyelash extensions and Demodex folliculorum, it is important to examine the existing body of research on this topic. The enhancement of eyelashes via cosmetics has been in practice for centuries but eyelashes, let alone eyelash extensions, have been minimally researched due to their unique and comparatively rare nature compared to other forms of hair. In fact, according to a peer-reviewed article written by Montreal University professors Sarah Aumond and Etty Bitton, the first study that comprehensively researched eyelashes was only conducted in 1969, where the "histology and cytochemistry of human lids and lashes" was initially analyzed. This experiment used the attraction between proteins to create a magnified model of the shape and structure of a human eyelash. ${ }^{3}$ Research regarding eyelashes has since passed through various stages in its development.

A large focus in eyelash research thereafter became the microorganisms and bacteria that inhabit eyelashes and cause health problems. The Research Center for Biomolecules and Biosystems sector of Chung-Ang University researched how the microbes on the eyelashes of patients with and without blepharitis, eyelid inflammation caused by bacterial infection, varied. To run their experiment, the scientists collected four eyelashes from each patient and examined the eyelashes under a microscope for Demodex folliculorum and other parasites. Once these parasites were removed, the foreign genetic material remaining on the eyelashes was matched to its bacterial name through a complex process known as pyrosequencing. The bacterium genera "Staphylococcus, Streptophyta, Corynebacterium, and Enhydrobacter, and a decrease of Propionibacterium were observed" in the eyelashes of patients that had an infection.

${ }^{13}$ Peer-reviewed journal Seminars in Ophthalmology corroborates the findings of Chung-Ang University in terms of bacteria on eyelashes. Using medical examination involving various intensive tests on eyelashes, the researchers not only similarly concluded that Staphylococcus can be found on eyelashes, but also bacterial overpopulation on eyelashes can occur. This could lead to Acute Staphylococcal blepharitis, which is evidently is not a desirable outcome, but a real possibility if standard hygienic precautions are not taken. ${ }^{4}$

Tangential to bacteria on eyelashes is parasites, or more specifically Demodex folliculorum, for the purposes of this paper. In an attempt to find the pathogenic role of Demodex folliculorum in blepharitis, Jingbo Liu, Hosam Sheha, and Scheffer C.G. Tseng of the Ocular Surface Research \& Education Foundation conduct an experiment with a procedure resembling that of the Chang-Ang University: top and bottom eyelashes of blepharitis patients were collected, sanitized, and viewed under a microscope. The Demodex folliculorum was manually counted under the microscope and the researchers were able to resolve that each eyelash of a blepharitis patient had the parasite. ${ }^{15}$ Despite having a different motive for research and a distinct procedure than all of the priorly mentioned experiments, The Department of Dermatology at Johns Hopkins University has ultimately confirmed the incidence of Demodex folliculorum on eyelashes. This experiment aimed to find suitable treatments for blepharitis and did so by directly measuring the symptoms of blepharitis on randomized patients with blepharitis. However, when analyzing the collected data, the Johns Hopkins scientists mentioned that "Demodex mites have also been considered a causative factor for blepharitis. The mites, which infest the eyelid margin around the lash follicles and sebaceous glands, may have a role in both anterior and posterior blepharitis". ${ }^{14}$ Therefore, several studies exist in the body of knowledge regarding eyelashes and the harm bacteria and Demodex folliculorum can cause from a health viewpoint.

The already dangerous risks that are associated with improper hygiene with natural eyelashes are amplified when eyelash extensions are involved. Amano, Yuki MD; Sugimoto, Yuka MD; and Sugita, Minoru MD, PhD are Graduate Students of Medicine from The Tokyo University who followed the medical history (consented) of 107 
women that did not have ocular disorders prior to the usage of eyelash extensions. The data they collected suggests that "The ocular disorders due to eyelash extensions included keratoconjunctivitis due to invasion of glue or removing agents in 64 patients, allergic blepharitis due to glues in 42 patients ( 4 of these patients developed both keratoconjunctivitis and allergic blepharitis), conjunctival erosion due to eyelid-fixing tapes in 3 patients, allergic blepharitis due to eyelid-fixing tapes in 1 patient, and subconjunctival hemorrhage due to compression during removal of extensions in 1 patient". ${ }^{1}$ The transition from no preexisting conditions to several health concerns following the usage of eyelash extensions outlines the danger this product presents. Researchers at the University of Utah expand upon the work from Tokyo University and present a hypothesis as to why eyelash extensions pose a large threat on ocular health. After synthesizing multiple studies through a meta-analysis approach, the researchers concluded that "the cause of these ocular responses and narrowed down the sources to the lash glue adhesive and to mechanical limitations of the lash extensions themselves". ${ }^{16}$ This explanation is valid when analyzing the properties of the chemicals in the eyelash extension glue, but is not supported by lab or statistical evidence, which weakens their hypothesis.

The correlation that specifically eyelash extensions have with Demodex folliculorum and bacteria compared to that of regular eyelashes has yet to be researched thoroughly. As of 2014, the US eyelash extension industry was worth $\$ 55$ million, and undoubtably that sum is larger now. ${ }^{3}$ With the increasingly popular preference of eyelash extensions over other eyelash cosmetics, consumers must be aware of the safest options available to them. Mink, sable, and silk eyelash extensions each have their own proponents and setbacks, but no information is available on which eyelash extension type is least prone to harboring Demodex folliculorum and other microbes. Consequently, as stated previously, one would be led to question "How does the composition of differing eyelash extension types impact the growth of bacterium that ultimately promotes the increase of the parasite Demodex folliculorum near the eye?"

\section{Methods}

When designing an experimental procedure to test the research question, there were inhibiting factors that prevented the experiment to be conducted in a similar fashion to the scientists in the literature review. The first of which was that it was merely unethical to endanger the students or faculty at a high school by collecting worn eyelash extensions and simply counting the Demodex folliculorum found on them, as many scientists did. Demodex folliculorum is a parasite, and one cannot not intentionally bring the species and observe its behavior at a school. Therefore, it was determined that observing bacterial growth on eyelash extensions, and then correlating the relationship between bacteria and Demodex folliculorum to ultimately determine the relationship between the type of eyelash extensions and Demodex folliculorum was a more viable option. The Coronavirus pandemic also played an instrumental role in the way the experiment was finally performed. Revisions of procedural drafts and preparatory steps had been finalized for the experiment to occur in a school lab, but schools closed nationwide before a significant amount of data was collected. Due to the quarantine measures that prevented accessibility to any school or University's labs and equipment, the experimental procedure priorly set up underwent several altercations to be safely conducted at home.

However, as initially planned, the research method that utilized is quasi-experimental, and there are several reasons as to why this is the case. The nature of a quasi-experimental experiment is to explore and explain possible answers to the proposed research question. The explore portion of the method is collecting primary data of bacterial growth on eyelash extensions, and the explain is connecting bacterial growth to Demodex folliculorum thereby explaining the relationship between eyelash extensions and Demodex folliculorum. The design of the experiment is evidently experimental, which is another characteristic of a quasi-experimental approach. Finally, a quasi-experimental experiment consists of a heavy usage of both primary and secondary data. As stated previously, the primary data collected will be derived from the experiment that is run, and the secondary data is the information that is collected from scholarly sources that will ultimately tie in Demodex folliculorum into the topic at hand.

The nationwide quarantine precautions led to personal purchases for the equipment required to run the experiment. A table enlisting all products and expenses is included for assistance in experiment replication. 
Table 1. Itemized list of materials

\begin{tabular}{|c|c|c|c|c|}
\hline $\begin{array}{l}\text { Item/Order } \\
\text { Number }\end{array}$ & Product Name & Company & Quantity & Cost \\
\hline $\begin{array}{l}\text { ORDER } \\
\# 1112\end{array}$ & $\begin{array}{l}\text { MINK Basic Eyelash Extension } \times 1 \\
\text { C / } 0.15 / 8 \mathrm{~mm}\end{array}$ & Bling Online Store & 1 pack & $\$ 9.99$ \\
\hline$\# 1112$ & $\begin{array}{l}\text { SILK Basic Eyelash Extension x } 1 \\
\text { C / } 0.15 \text { / 8mm }\end{array}$ & Bling Online Store & 1 pack & $\$ 10.99$ \\
\hline \#1112 & $\begin{array}{l}\text { SABLE Basic Eyelash Extension } \mathrm{x} \\
1 \\
\mathrm{C} / 0.15 / 8 \mathrm{~mm}\end{array}$ & Bling Online Store & 1 pack & $\$ 28.99$ \\
\hline \#776382 & $\begin{array}{l}\text { Nutrient Broth, Prepared Media } \\
\text { Bottle, } 125 \mathrm{~mL} \text {, Plastic Bottle }\end{array}$ & Amazon & 1 bottle & $\$ 10.90$ \\
\hline \#002 & Barbicide Table Manicure Jar & Amazon & $1 \mathrm{jar}$ & $\$ 12.33$ \\
\hline$\# 155557$ & STAPH. EPIDERMIDIS BROTH & Carolina & 1 tube & $\$ 11.95$ \\
\hline$\# 155068$ & $\begin{array}{l}\text { BACTERIA, ESCH COLI K12 } \\
\text { BROTH }\end{array}$ & Carolina & 1 tube & $\$ 11.95$ \\
\hline $\begin{array}{l}\text { \#CE- } \\
\text { TTBSB06 }\end{array}$ & $\begin{array}{l}\text { Giant Test Tubes (Baby Soda Bot- } \\
\text { tles), } 6 \text { Pack }\end{array}$ & HomeScienceTools & 1 kit & $\$ 5.00$ \\
\hline $\begin{array}{l}\text { \#CE- } \\
\text { MP010UL }\end{array}$ & Micropipette, $10 \mathrm{ul}$ & HomeScienceTools & 1 item & $\$ 23.50$ \\
\hline $\begin{array}{l}\text { \#CE- } \\
\text { STIRROD }\end{array}$ & Stirring Rod, glass , 6" & HomeScienceTools & 1 item & $\$ 5.00$ \\
\hline $\begin{array}{l}\text { \#BE- } \\
\text { AGARNBT }\end{array}$ & $\begin{array}{l}\text { Nutrient Agar, Prepared Media Bot- } \\
\text { tle, } 125 \mathrm{ml}\end{array}$ & HomeScienceTools & 1 bottle & $\$ 7.80$ \\
\hline $\begin{array}{l}\text { \#BE- } \\
\text { PETRI20 }\end{array}$ & $\begin{array}{l}\text { Petri Dishes, polystyrene, } 90 \times 15 \\
\mathrm{~mm}, 20 \text { pack }\end{array}$ & HomeScienceTools & 1 pack & $\$ 7.05$ \\
\hline $\begin{array}{l}\text { \#BE- } \\
\text { INOCUL }\end{array}$ & $\begin{array}{l}\text { Inoculating Needle with Looped } \\
\text { End }\end{array}$ & HomeScienceTools & 1 item & $\$ 3.60$ \\
\hline$\# 868452$ & Ardell Brush-on Lash Adhesive & CVS Pharmacy & 1 item & $\$ 5.49$ \\
\hline \#230336 & Beauty 360 Slant Tweezers & CVS Pharmacy & 1 item & $\$ 2.79$ \\
\hline
\end{tabular}

Alongside the equipment listed in the table above, items including scissors, distilled water, gloves, and other supplies that help maintain aseptic technique were necessary for experimentation are readily available at any convenience store. The most quintessential materials for this experiment were the eyelash extensions, and to understand the results that are yielded, the differences between the types of eyelash extensions must be understood.

The most sought after are the eyelash extensions that are derived from mink hairs-mink eyelash extensions. Mink eyelash extensions are often deemed the most comfortable and well-worth their moderately affordable price (refer to Table 1), as stated by the well-recognized eyelash extension supplier Borboleta Beauty. ${ }^{19}$ As the name suggests, mink eyelash extensions "come from the Mink tail, usually coming from either the Siberian Mink or the Chinese Mink". ${ }^{19}$ Legally required sanitization laws, specifically the regulations related to cosmetics from Title 21 of the Code of Federal Regulation, now ensure that unhygienic traces a mink may have left are removed before these eyelash extensions are applied. ${ }^{5}$ Unfortunately, according to the Advertising Standard Authority of Ireland, it has been found that the hairs obtained from the minks may not have been ethical in the past. Yet, for safety and ethical reasons, the mink eyelashes extensions (and sable and silk) used for the purposes of this study were from BlingLash New York, an eyelash extension supplier that claims to prioritize antimicrobial practices and animal rights. ${ }^{5}$ 
Another type of eyelash extension that is commonly fashioned is called sable eyelash extensions. More formally known as Siberian sable fur eyelash extensions, this type of eyelash extension is known for its extreme elasticity, lightness, and overall natural look. BeautyGarde, a website created by cosmetic experts, elaborates to users when stating that sable eyelash extensions are "even more fine and fluffy than mink. It's [sable] the lightest possible material available for extensions". ${ }^{19}$ As a result of their uniqueness, sable eyelashes are both the rarest and the most expensive at salons that provide customers with eyelash extensions and are mainly recommended to people that have naturally thin eyelashes. Sable eyelash extensions are composed of PBT fiber, ${ }^{5}$ which is a synthesized polymer, ${ }^{9}$ or a large molecule formulated by the combining of smaller organic molecules. Sable eyelash extensions are also the most expensive eyelash extensions, as seen in Table 1.

The final main type of eyelash extension is composed of silk "from the cocoons of the silkworm" and is commonly referred to as silk eyelash extensions. ${ }^{12}$ Borboleta Beauty regards silk eyelash extensions as the "midweight type of lash extensions and are finer and more flexible than the synthetic type which makes their retention slightly better". ${ }^{19}$ Generally, silk eyelash extensions are favored by those who are getting eyelash extensions for the first time or are unsure of what eyelash look suits them best. ${ }^{18}$ These eyelash extensions are the type that give the wearer the most dramatic look of the three and are a median price for eyelash extensions (Table 1). ${ }^{5}$ However, the main advantage that silk eyelash extensions provide is their durability. Annie Le, an eyelash extension technician from New York, claims that silk eyelash extensions are "less likely to come off the band when you're removing them or cleaning off the glue," which also promotes users to clean their eyelash extensions more frequently. ${ }^{12}$

After analyzing the composition and qualities of each eyelash extension type, the derived hypothesis was that sable eyelash extensions would yield the least bacterial growth, and consequently Demodex folliculorum in real life, and mink eyelash extensions would lead to the most growth. This conclusion was approached because Sable eyelash extensions were the most expensive by a significant amount, and because silk and mink eyelash extensions are derived from real biodiversity, while sable eyelash extensions are synthesized from man-made plastics.

The sable eyelash extensions arrived after the mink and silk extensions, but the same procedure was used to test all three eyelash extensions for bacterial growth. Prior to the start of the experiment, the contents of the nutrient agar bottle were melted and poured into petri dishes. Nutrient agar is a solid medium that can support a plethora of bacterium to grow due to its high concentration of nutrients. ${ }^{2}$ The petri dishes were stored in the fridge until the agar within was solidified and ready for bacterial cultivation.

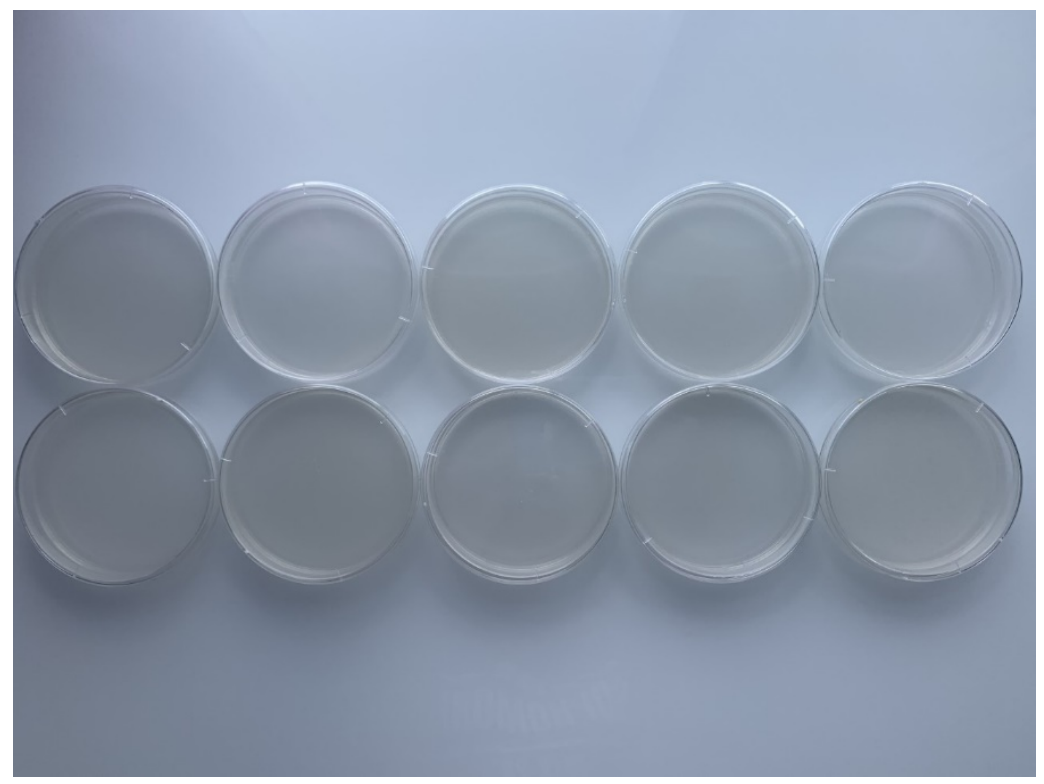

Figure 1. Nutrient agar in petri dishes 
Thereafter, sterilization of the tweezers, scissors, and eyelash extensions using Barbicide. 2oz of barbicide concentrate to $32 \mathrm{oz}$ of cold distilled water for 10 minutes occurred. Eyelash extension technicians utilize barbicide to disinfect all materials because it is a quick, effective, and safe method to clean all materials. ${ }^{25}$ All surfaces lab materials would come in contact with were cleaned using Clorox or bleach.

After cleaning all surfaces, the eyelash extensions needed to be assembled. The eyelash extensions were sold as strips to be cut for application, so the glue had to dry for five minutes before cutting each eyelash extension type into four three-centimeter pieces. As previously stated, the glue was found to have an impact on the bacteria accumulated on the eyelash, so each piece of the extension had $2 \mathrm{~mL}$ of the glue applied. While the glue dried, the test tubes were labeled as follows in table 2 .

Table 2. Test Tubes

\begin{tabular}{|c|l|c|l|}
\hline $\begin{array}{c}\text { Test Tube } \\
\text { Number }\end{array}$ & \multicolumn{1}{|c|}{ Eyelash Extension Type } & Nutrient Broth & \multicolumn{1}{|c|}{ Bacteria } \\
\hline$\# 1$ & Mink Eyelash Extensions & Yes & Staphylococcus epidermidis \\
\hline$\# 2$ & Mink Eyelash Extensions & Yes & Escherichia coli \\
\hline$\# 3$ & Silk Eyelash Extensions & Yes & Staphylococcus epidermidis \\
\hline$\# 4$ & Silk Eyelash Extensions & Yes & Escherichia coli \\
\hline$\# 5$ & Mink Eyelash Extensions & Yes & N/A \\
\hline$\# 6$ & Mink Eyelash Extensions & No & N/A \\
\hline$\# 7$ & Sable Eyelash Extensions & Yes & Staphylococcus epidermidis \\
\hline$\# 8$ & Sable Eyelash Extensions & Yes & Escherichia coli \\
\hline
\end{tabular}

Each eyelash extension type was to be tested with both S. epidermidis and E. coli, and two controls were present as well. Because test tube \#5 had nutrient broth but no bacteria, any growth in the test tube would be a result of contamination of bacteria outside of the nutrient broth. Test tube \#6 had neither nutrient broth nor bacteria, so growth in this test tube would indicate that the eyelash extensions themselves were a source of contamination.

All test tubes excluding test tube \#6 had $20 \mathrm{~mL}$ of nutrient broth, and all except tubes \#5 and \#6 had $20 \mathrm{ul}$ of their respective bacteria inserted through a micropipette. This made the bacterial wash's bacteria to nutrient broth ratio 1:1000. After injecting the nutrient broth and liquid into the test tubes, the designated eyelash extensions were placed in the test tubes and sealed. At room temperature, Staphylococcus epidermidis and E. coli take around 72 hours to grow, and while they were growing, the test tubes were rotated every hour to simulate the motion that eyelash extensions undergo on a person's eye.

After 72 hours, a tweezer was used to remove the eyelash extensions out of the test tube onto the nutrient agar plates that were created at the start of the experiment. By extracting the eyelash extensions that were covered in growing bacteria and placing them on a new growth medium, one can quantifiably analyze how much of the bacteria became trapped in the eyelash extensions. The nutrient agar plates were labeled in correspondence to the test tubes as follows in table 3 . 
Table 3. Plates

\begin{tabular}{|l|l|l|}
\hline Plate Number & \multicolumn{1}{|c|}{ Eyelash Extension Type } & \multicolumn{1}{c|}{ Bacteria } \\
\hline$\# 1$ & Mink Eyelash Extensions & Staphylococcus epidermidis \\
\hline$\# 2$ & Mink Eyelash Extensions & Escherichia coli \\
\hline$\# 3$ & Silk Eyelash Extensions & Staphylococcus epidermidis \\
\hline$\# 4$ & Silk Eyelash Extensions & Escherichia coli \\
\hline$\# 5$ & Mink Eyelash Extensions & N/A \\
\hline$\# 6$ & Mink Eyelash Extensions & N/A \\
\hline$\# 7$ & Sable Eyelash Extensions & Staphylococcus epidermidis \\
\hline$\# 8$ & Sable Eyelash Extensions & Escherichia coli \\
\hline
\end{tabular}

Following three days of growth on the nutrient agar, the bacteria on the eyelash extensions had formed measurable colonies. Once these results had been documented, the test tubes and the plates were bleached to kill any remaining bacterium.

\section{Results}

If the circumstances had been different and this experiment were to occur in a school lab, then multiple runs would be able to deduce a margin of error with this experiment. With this calculated margin of error, one could determine whether the amount of colonies produced by each eyelash extension type was statistically significant or not. However, this experiment was conducted once at home, so the results are based off of the number of colonies seen.

Within this experiment, there were two mediums upon which the bacteria were cultivated. The first was nutrient broth, or the liquid growing medium.

A

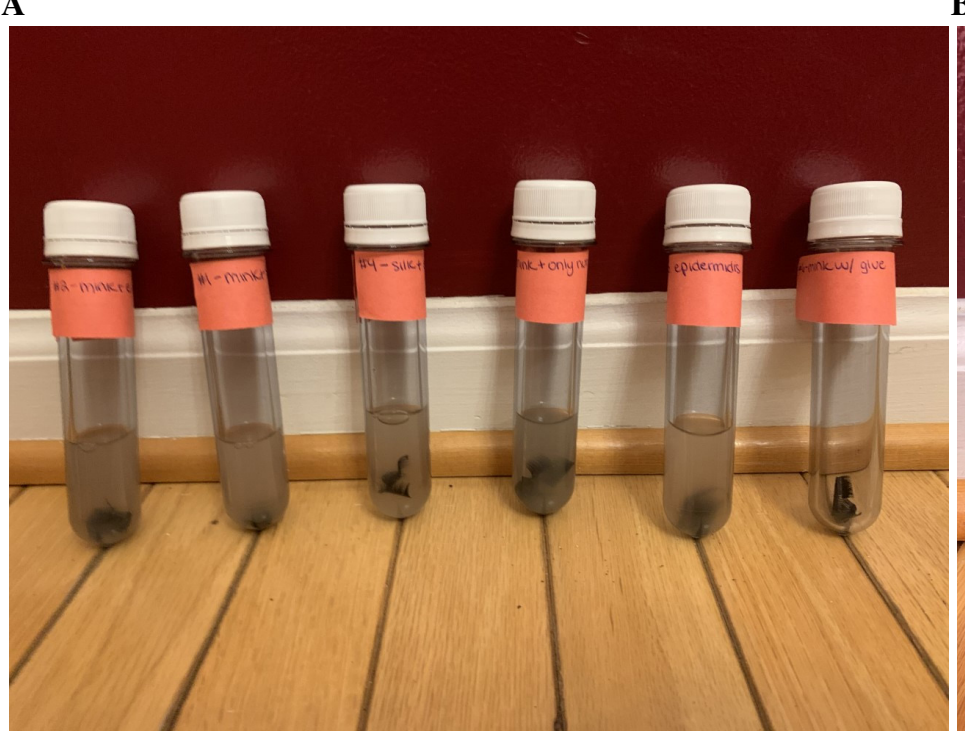

B

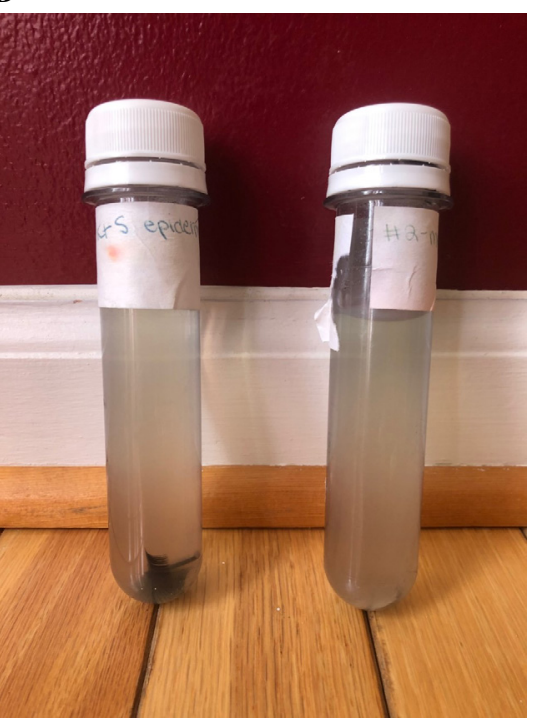

Figure 2. Test Tubes 1-6 pictured in A and 7-8 in B after 72 hours of bacterial growth.

The growth in the nutrient broth was not a quantifiable amount, which is why another growth medium had to be used. While the bacterium was growing inside of the test tubes, the eyelash extensions were meeting the colonies. After the growth in nutrient broth, the eyelash extensions were extrapolated and placed in the nutrient agar plates. 


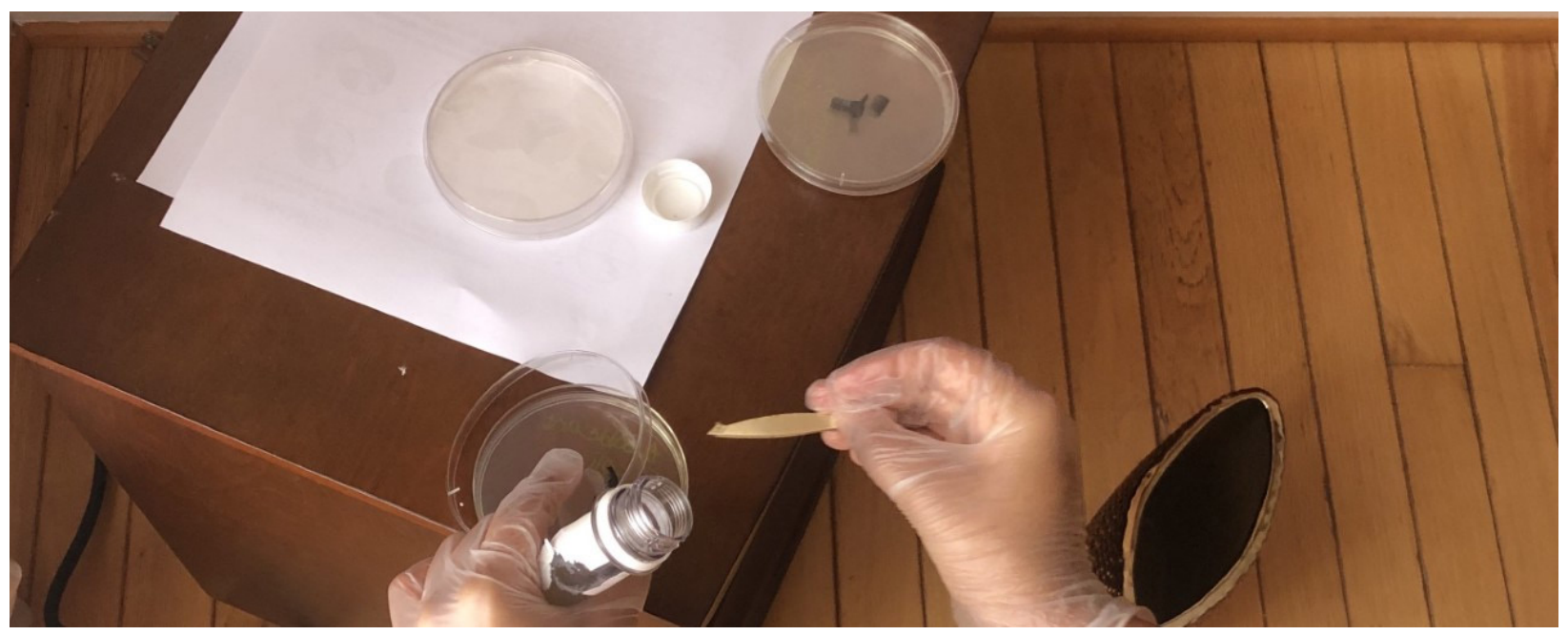

Figure 3. The eyelash extensions are being removed from the test tube to the plate using a tweezer. The hinge-method is being utilized to open the plate to minimize contamination via column of vertical air.

The eyelash extensions were directly taken from the nutrient broth with bacteria into the clean nutrient agar plates. The bacteria that the eyelash extensions had trapped during the time in the test tubes were transferred with the eyelash extensions, and the presence of the bacteria was proved with the growth of the colonies coming from the eyelash extensions. The following data table and pictures reveal the number of colonies recorded on each plate after 72 hours of growth at $75^{\circ} \mathrm{F}$.

Table 3. Growth on Plates

\begin{tabular}{|l|l|l|l|}
\hline Plate Number & \multicolumn{1}{|c|}{ Eyelash Extension Type } & \multicolumn{1}{c|}{ Bacteria } & \multicolumn{1}{c|}{ Colonies } \\
\hline$\# 1$ & Mink Eyelash Extensions & Staphylococcus epidermidis & 572 \\
\hline$\# 2$ & Mink Eyelash Extensions & Escherichia coli & 83 \\
\hline$\# 3$ & Silk Eyelash Extensions & Staphylococcus epidermidis & 399 \\
\hline$\# 4$ & Silk Eyelash Extensions & Escherichia coli & 64 \\
\hline$\# 5$ & Mink Eyelash Extensions & N/A & 216 \\
\hline$\# 6$ & Mink Eyelash Extensions & N/A & 0 \\
\hline$\# 7$ & Sable Eyelash Extensions & Staphylococcus epidermidis & 57 \\
\hline$\# 8$ & Sable Eyelash Extensions & Escherichia coli & 48 \\
\hline
\end{tabular}


A

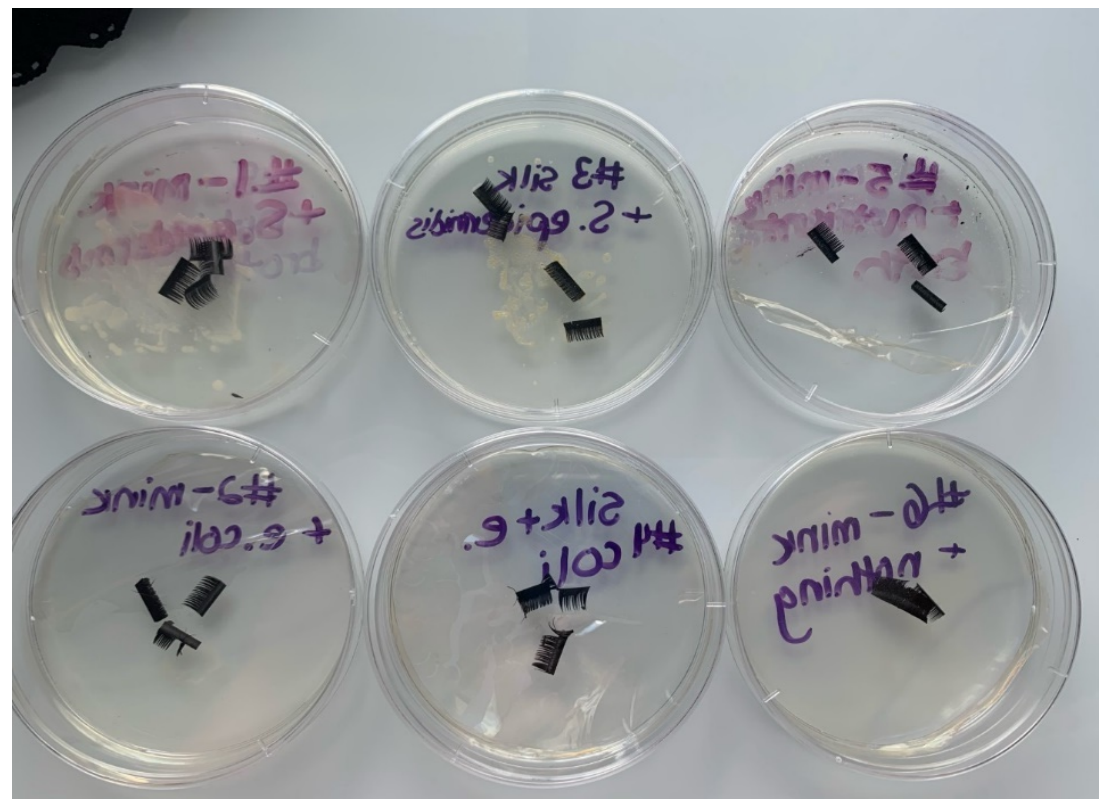

B

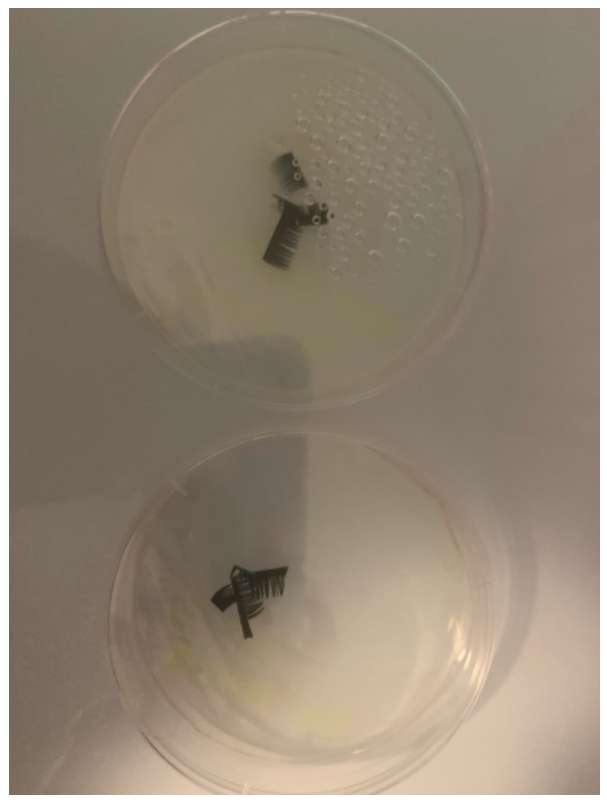

Figure 4. Plates 1-6 pictured in A and plates 7-8 in B. Lighting and picture is unfocused on the right (B), but the quantity of colonies on each plate was clear in-person, and is listed in Table 3.

\section{Discussion}

The eyelash extension that was correlated with the least growth of colonies would ultimately lead to the least growth of the eyelash parasite Demodex folliculorum. As indicated by the data collected, sable eyelash extensions had the least colony growth, with 57 Staphylococcus epidermidis colonies and 48 Escherichia coli colonies, whereas mink eyelash extensions had the most colony growth, with 572 and 83 colonies, respectively. Therefore, it appears that the safest eyelash extension type in terms of bacterial accumulation is sable eyelash extensions.

Through the results of the experiment, one can deduce that the hypothesis was correct. The sable eyelash extensions did in fact grow the least colonies, while mink eyelash extensions grew the most. Due to the positive correlation between Escherchia coli and Staphylococcus epidermidis with E. coli, it can be concluded that sable eyelash extensions would also promote the least growth of Demodex folliculorum. This is most likley a result of the artificial composition of sable eyelash extensions that is less appealing to microorganisms compared to the naturallyexisting and thin structure of mink eyelash extensions.

The body of knowledge has indicated that bacteria, especially those that have traits similar to Staphylococcus epidermidis and Escherichia coli, can serve as a vector for Demodex folliculorum. This essentially means that bacteria and Demodex folliculorum have a mutually beneficial relationship, or that one helps the other sustain. When presented with the three options of mink, silk, and sable eyelash extensions, the user would most likely wish to avoid the choice that leads to more growth of Demodex folliculorum, commonly reknowned as "eyelash lice." Considering the data in Table 3 provides evidence that this eyelash extension has the least detected bacterium, and the fact that less bacterium would lead to less Demodex folliculorum, it can be concluded that sable eyelash extensions would have less Demodex folliculorum than its counterparts.

It may be noted that plate \#5 had noticable colony growth, even though it only had nutrient broth, which means that it should have had zero colony growth -- resembling that of test tube \#6. It may be unclear in the picture, but the colonies that grew on plate \#5 were S. epidermidis colonies, which indicates contamination. While it is hard to discern whether the contamination occurred because the nutrient broth became exposed to S. epidermidis or the 
tweezers were not properly sanitized before switching tubes, it is clear that this contamination still lead to less colonies than the eyelash extensions tested prior and no contamination was done to tube \#6 and afterwards. This increases to the confidence that this small error did not impact what the derived results are.

The resuls that were collected are also supported by current research. Both mink and silk are composed of real organic material from an organism, which means that their structures are heavily reliant on Carbon. The sable eyelash extensions, on the other hand, are made of a man-made polymer known as PBT fiber. Although PBT fiber, is also composed of organic materials, because it is not naturally occuring, other organisms would not be as attracted to it. Hence, both the data and the existing body of knowledge indicate that sable eyelashes will promote the least growth of Demodex folliculorum.

\section{Conclusion}

As predicted, mink eyelashes lead to the most growth of Demodex folliculorum while sable eyelashes are the safest option. Throughout the research on the current body of knowledge and the trials, sable was proven that the healthiest to dangerous eyelash extensions are sable, silk, and mink eyelash extensions.

If given more time to conduct research, or if the pandemic had not taken place, there are several procedures that would have been carried out differently. Of course, all that is under the limitations are aspects that would have been fixed or attempted to solve if given more time. However, another take on this experiment is to do the same experiment with more bacteria. While conducting initial research, other bacterium, most notibly Bacillus oleronius and Streptococcus pyogenes, were found to be closely related to Demodex folliculorum. ${ }^{13}$ Observing how their colonies would grow after coming in contact with eyelash extensions would certainly provide a new perspective to the results yielded from this experiment. With extra time and the opportunity to work in a science laboratory, one could also directly moniter Demodex folliculorum on eyelash extensions and be able to to cut the middle bacteria step, just as scientists do.

There are a handful of future directions to prevent or minimize the growth of Demodex folliculorum in eyelash extensions. The first of which would be to raise awareness about the stigma that thorughly washing eyelash extensions would weaken the extensions and induce them to come off more quickly. The fear that people have prevents them from being hygenic and certainly promotes microorganisms such as Demodex folliculorum to prevail. The Demodex in turn leads to a plethora of unwanted health concerns, including Blepharitis and Acute Staphylococcal diseases. ${ }^{9}$ Another suggestion is to increase the stregnth of the eyelash extension glue and substitute the ingredients Cyanoacrylate and Formaldehyde. These active ingredients have been found to be correlated to the presence of Demodex folliculorum, and a stronger glue would also aid in removing the concern of the eyelash extensions coming undone with diligient washing. It is ideal to prevent Demodex folliculorum to live in eyelash extensions, but treatments to minimize and eradicate the parasites already existant in the eyelash extensions are also quite shallow and weak, so it is recommend that further research be conducted by the medical community in finding treatments for Demodex folliculorum growth on eyelash extensions.

This research contributes to both the cosmetics body of knowledge as well as the biological body of knowledge. Although the relationship between eyelashes and Demodex folliculorum has been researched in the past, the quantitiative correlation between the varying types of eyelash extensions and Demodex folliculorum was unknown to the community. With this new-found knowledge, scientists are informed of the materials that correlate to the growth of these microorganisms, and users of eyelash extension are aware of which of their choices is the least harmful.

\section{Limitations}

Several limitations arose througout this experimental procedure. As already outlined, multiple trials of the experiment to determine a margin of error could not be conducted and one of the controls became contaminated. Another limitation 
is that the experiments done on the sable eyelash extensions were done at a different time than the mink and silk eyelash extensions due to the cautious shipping during the pandemic. Although the room, room temperature, equipment, and all other variables that could be controlled were constant, there may have been some factors out of one's control that may have lead to the yielded results. Yet another limitation to the experiment is the temperature and humidity the bacteria was grown at. Both Escherichia coli and Staphylococcus epidermdis's optimal temperature is 37 degrees Celcious and in a humid environment, but instead they were cultivated at approximately 25 degrees Celcius in a comparitively dry room. In fact, the dryness of the room lead the agar to slightly peel, which is depicted in Figure 4. At school, an incubator would have been readily availible, but unfortunately an incubator was not affordable, so the experiment was operated at room temperature and humidity.

\section{Acknowledgments}

I would like to express my immense appreciation to my AP Research teacher, Mrs. Webb, for her valuable and constructive suggestions throughout the development of my research, and Mrs. Vought for the guidence she provided in the formatting and citations of this paper. Their readiness to set aside their time so kindly has been truly appreciated. Special thanks to my parents whom were willing to purchase the equipment necessary for this experiment and provide assistance in any way possible. The pandemic certainly presented unique obstacles in the research proccess, but I am very grateful I had their constant support in these unprecedented times.

\section{References}

1. Amano Y, Sugimoto Y, Sugita M. Ocular disorders due to eyelash extensions. Cornea. 2012;31(2):121125. doi:10.1097/ico.0b013e31821eea10

2. Aryal S, Emmanuel O, Anusree, Onuchi OA, David, Bradbury S, Veyan L, Aeny TN, Sharma R, C K, et al. Nutrient agar: composition, preparation and uses. Microbiology Info.com. 2018 Sep 26 [accessed 2020 May 25]. https://microbiologyinfo.com/nutrient-agar-composition-preparation-and-uses/

3. Aumond S, Bitton E. The eyelash follicle features and anomalies: A review. Journal of Optometry. 2018 [accessed 2020 May 25]. https://www.ncbi.nlm.nih.gov/pmc/articles/PMC6147748/\#bib0950

4. Bernardes TF, Bonfioli AA. Blepharitis. Seminars in Ophthalmology. 2010 [accessed 2020 May 24];25(3):79-83. doi:10.3109/08820538.2010.488562

5. Bling Online Store. Bling Online Store. [accessed 2020 May 26]. https://www.blingonlinestore.com/

6. Center for Food Safety and Applied Nutrition. Regulations related to cosmetics in 21 CFR. U.S. Food and Drug Administration. 2018 Jul 3 [accessed 2020 May 25]. https://www.fda.gov/cosmetics/cosmetics-laws$\underline{\text { regulations/regulations-related-cosmetics-title-21-code-federal-regulations-21-cfr }}$

7. Clifford CW, Fulk GW. Association of diabetes, lash loss, and Staphylococcus aureus with infestation of eyelids by Demodex folliculorum (Acari: Demodicidae). Journal of Medical Entomology. 1990 Jul 30 [accessed 2020 May 25]. https://www.ncbi.nlm.nih.gov/pubmed/2388222

8. Huismans H. Demodex folliculorum. Klinische Monatsblatter fur Augenheilkunde. 1988 Sep [accessed 2020 May 25]. https://www.ncbi.nlm.nih.gov/pubmed/3236736 
9. Jade Coats OD. Blog: A case of Demodex infestation with eyelash extensions. Optometry Times. 2019 Apr 24 [accessed 2020 May 25]. https://www.optometrytimes.com/article/blog-case-demodex-infestation-eyelash-extensions

10. Kwartang A. Eyelash extensions for beginners: everything you need to know. Cosmopolitan. 2019 Aug 16 [accessed 2020 May 25]. https://www.cosmopolitan.com/style-beauty/beauty/a3915095/eyelash-extensions-for-beginners/

11. Lacey N, Kavanagh K, Tseng SCG. Under the lash: Demodex mites in human diseases. The Biochemist. 2009 Aug 1 [accessed 2020 May 25]. https://www.ncbi.nlm.nih.gov/pmc/articles/PMC2906820/

12. Le A. Silk vs mink lashes - which is better? Mademoiselle Lash. 2019 Dec 10 [accessed 2020 May 25]. https://www.mademoisellelash.com/blogs/all-about-lashes/silk-or-mink-false-eyelashes-which-is-better

13. Lee SH, Oh DH, Jung JY, Kim JC, Jeon CO. Comparative ocular microbial communities in humans with and without Blepharitis. Investigative Opthalmology \& Visual Science. 2012 [accessed 2020 May 24];53(9):5585. doi:10.1167/iovs.12-9922

14. Lindsley K, Matsumura S, Hatef E, Akpek EK. Interventions for chronic blepharitis. The Cochrane database of systematic reviews. 2012 May 16 [accessed 2020 May 25]. https://www.ncbi.nlm.nih.gov/pmc/articles/PMC4270370/

15. Liu J, Sheha H, Tseng SCG. Pathogenic role of Demodex mites in Blepharitis. Current Opinion in Allergy and Clinical Immunology. 2010 Oct [accessed 2020 May 25]. https://www.ncbi.nlm.nih.gov/pmc/articles/PMC2946818/

16. Masud M, Moshirfar M, Shah TJ, Gomez AT, Avila MR, Ronquillo YC. Eyelid cosmetic enhancements and their associated ocular adverse effects. Medical hypothesis, discovery \& innovation ophthalmology journal. 2019 [accessed 2020 May 25]. https://www.ncbi.nlm.nih.gov/pmc/articles/PMC6592309/

17. McBride C. 'Cruelty free' false lashes came from minks - ASAI Upholds Complaint Against Irish Beauty Company. independent. 2018 Oct 31 [accessed 2020 May 25]. https://www.independent.ie/style/beauty/cruelty-free-false-lashes-came-from-minks-asai-upholds-complaint-against-irish-beautycompany-37478203.html

18. McIntyre A. The difference between mink, silk, \& real fur eyelashes. Sugarlash PRO. [accessed 2020 May 25]. https://sugarlashpro.ca/blogs/news/what-s-the-difference-between-mink-silk-and-real-fur-lashes

19. Mink, silk and faux mink extensions - what's the difference? Borboleta Beauty. [accessed 2020 May 25]. https://borboletabeauty.com/blogs/news/mink-silk-and-faux-mink-extensions-what-s-the-difference

20. Namvar AE, Bastarahang S, Abbasi N, Ghehi GS, Farhadbakhtiarian S, Arezi P, Hosseini M, Baravati SZ, Jokar Z, Chermahin SG. Clinical characteristics of Staphylococcus epidermidis: a systematic review. GMS Hygiene and Infection Control. 2014 Sep 30 [accessed 2020 May 25]. https://www.ncbi.nlm.nih.gov/pmc/articles/PMC4184040/ 
21. Owusu-Darko R, Allam M, Mtshali S, Ismail A, Buys EM. Draft genome sequence of Bacillus oleronius DSM 9356 isolated from the termite Reticulitermes santonensis. Genomics Data. 2017 Mar 24 [accessed 2020 May 25]. https://www.ncbi.nlm.nih.gov/pmc/articles/PMC5374852/

22. Reusch W. Aldehydes and ketones. Carbonyl Reactivity. 2013 May 5 [accessed 2020 May 26]. https://www2.chemistry.msu.edu/faculty/reusch/VirtTxtJml/aldket1.htm

23. RTP company. [accessed 2020 May 25]. https://www.rtpcompany.com/products/product-guide/polybutylene-terephthalate-pbt/

24. Shanmugam S, Wilkinson M. Allergic contact dermatitis caused by a cyanoacrylate-containing false eyelash glue. Contact Dermatitis. 2012;67(5):309-310. doi:10.1111/cod.12000

25. The Lash Professional. How to sterilize your lash extension tools. The Lash Professional. 2018 Apr 25 [accessed 2020 May 25]. https://www.thelashprofessional.com/business/sterilize-your-lash-extension-tools/

26. The optometrist's eyelash extension care guide to safer, healthier and happier eyes. We Love Eyes. 2017 Oct 8 [accessed 2020 May 25]. https://www.weloveeyes.com/blogs/news/the-eyelash-extension-care-guide

27. Ultimate guide to eyelash extensions: what are they, types \& styles. [accessed 2020 May 25]. https://beautygarde.com/ultimate-guide-to-choosing-the-best-eyelash-extensions/

28. What are side effects of eyelash extensions? Healthline. [accessed 2020 May 24]. https://www.healthline.com/health/eyelash-extension-side-effects\#takeaway 